\title{
Evaluation of Serum Vitamin D: An Institutional Study in Mumbai
}

\author{
Khanna $\mathrm{SS}^{* 1}$, Parulekar $\mathrm{N}^{2}$, Dhaimade $\mathrm{PA}^{3}$ and Gharpure $\mathrm{AS}^{4}$ \\ ${ }^{1}$ Department of Oral Medicine \& Radiology, Member National Academy of Medical Sciences, Nair Hospital Dental \\ College, Mumbai, India \\ ${ }^{2}$ Orthopedic Surgeon, Department of Orthopedics, Wockhardt Hospital, Mumbai, India \\ ${ }^{3}$ Former Intern, Nair Hospital Dental College, Mumbai, India \\ ${ }^{4}$ Private Practitioner, Mumbai, India
}

${ }^{*}$ Corresponding author: Khanna SS, Department of Oral Medicine \& Radiology, Member National Academy of Medical Sciences, Nair Hospital Dental College, Mumbai, Tel: 9892662345, E-mail: sunalikhanna@gmail. com

Citation: Khanna SS, Parulekar N, Dhaimade PA, Gharpure AS (2017) Evaluation of Serum Vitamin D: An Institutional Study in Mumbai. J Nutr Health Sci 4(3): 301. doi: 10.15744/2393-9060.4.301

Received Date: September 05, 2015 Accepted Date: November 27, 2017 Published Date: November 30, 2017

\begin{abstract}
Aim: The aim of the study was to evaluate the levels of serum $25(\mathrm{OH}) \mathrm{D}$ levels in patients to identify the average vitamin $\mathrm{D}$ levels, the incidence of insufficiency and deficiency amongst the patients reporting to a dental hospital in Mumbai.

Methods: Serum 25(OH)D levels of 100 randomly selected patients who reported to Nair Hospital Dental College in Mumbai were assessed to evaluate the vitamin D status. Serum levels below $10 \mathrm{ng} / \mathrm{mL}$ were categorized as "deficiency "and between $29-11 \mathrm{ng} / \mathrm{mL}$ were categorized as "insufficiency" of vitamin D.

Participants: 100 randomly selected patients reporting to the department of oral medicine and radiology on an outpatient basis.

Results: $52 \%$ of the individuals were identified to have insufficiency of vitamin $\mathrm{D}, 61.5 \%$ of the subjects who were identified with insufficiency were females. $13 \%$ of the total patients were identified with deficiency and $61.5 \%$ of those were females. The deficiency was seen to be seen majorly in age group above 40 years in females.

Conclusion: In spite of Mumbai being in the latitudes offering adequate exposure to sun, over half of the subjects in the study were identified with insufficiency of vitamin $\mathrm{D}$. The fraction of females was higher both in the insufficiency and deficiency and this may be regarded to various social customs prevalent in the country.
\end{abstract}

Keywords: Vitamin D; 25(OH)D; Insufficiency; Deficiency

\section{Introduction}

Not long ago, Vitamin D, the "sun shine" vitamin was considered inconsequential due to its wide availability and lack of research about the intricate role it plays in the various metabolic activities in the human body. However, with the advent of scientific research, a renewed interest has evolved in the role of Vitamin D3, both as a preventive and also a therapeutic modality for battling an array of disorders ranging from prevention of cancer, cardio vascular diseases, chronic illnesses like diabetes mellitus and multiple sclerosis; to dental problems such as prevention of caries, reduction in severity of periodontal diseases, and even prevention of premature alveolar bone loss [1-4]. It is now well established that Vitamin D plays an extremely important role in the metabolism of calcium thus having an important influence on the bones and that deficiency of vitamin D causes rickets in children and osteomalacia in adults. It also has an effect on the overall bone density, strength. The relationship of vitamin $\mathrm{D}$ with temporomandibular disorders is also being explored.

A number of non-skeletal benefits of vitamin D came to light with the discovery of vitamin D receptors and the 1a-hydroxylase enzyme in multiple tissues, including cells of the pancreas, immune system, macrophages, vascular endothelium, stomach, epidermis, colon, and placenta [5].

Measurement of serum 1,25(OH)2D concentrations is not used to evaluate the Vitamin D status of an individual. 25(OH)D is the pro hormone and the most stable metabolite of vitamin D in human serum. Since it has a half-life of about 3 weeks, it is one of the most appropriate metabolite to assess the serum vitamin D levels. Besides plasma 25(OH)D, calcidiol (a summation of D3 and D2 
forms) is also a reliable marker of vitamin D status of an individual. The various investigations such as radioimmunoassay (RIA), enzyme linked immunosorbant assay (ELISA), chemiluminescence immunoassay and protein binding assays are used in routine testing of $25(\mathrm{OH}) \mathrm{D}$ in laboratories. The most widely accepted reference method remains LCTMS (liquid chromatography tandem mass spectrometry). However it is not used commercially as it is tedious, time consuming and expensive [6].

It becomes extremely difficult to exactly define the values for deficiency that must be accepted universally because to the fact that vitamin D is synthesized in the body. Its levels depend primarily on the exposure of bare skin to the UV rays of the sun not on dietary sources. Besides the differences due to location and latitude, the differences in the melanin content of the skin are important variables in the synthesis of vitamin $\mathrm{D}$ in the human body $[7,8]$. Even so different investigators have given different cutoff values for deficiency. Historically, vitamin D deficiency was always identified by the presence of bone disease, either rickets or osteomalacia. Today we know, that bone disease is caused by vitamin D (serum 25(OH)D) values below $10 \mathrm{ng} / \mathrm{mL}$. Therefore serum 25(OH)D below $10 \mathrm{ng} / \mathrm{mL}$ is considered vitamin $\mathrm{D}$ deficiency.

A number of studies have tried to identify the optimum vitamin $\mathrm{D}$ levels and one of the functional definition of the same is the 25(OH)D level that maximally suppresses PTH secretion. Multiple cross sectional examinations that explored the relationship between serum PTH and 25(OH)D levels demonstrated a plateau in suppression of PTH when the 25(OH)D level reaches approximately $30 \mathrm{ng} / \mathrm{mL}$. This was used as a rationale to select the value of " $30 \mathrm{ng} / \mathrm{mL}$ " as a cutoff value. Consequently serum $25(\mathrm{OH}) \mathrm{D}$ levels above $10 \mathrm{ng} / \mathrm{mL}$ but below $30 \mathrm{ng} / \mathrm{mL}$ are identified as "insufficiency" [9]. Since India has a tropical environment, adequate exposure to the sun is expected and corresponding adequate average vitamin $\mathrm{D}$ levels are expected. However a number of studies have shown that deficiency and insufficiency of vitamin D is prevalent in all age groups all over the country, despite temperature, food habit and lifestyle variations [10-12]. Studies carried out all over the country estimate that the prevalence of Vitamin D deficiency is varied (50-90\%) [13].

Vitamin D deficiency is often silent initially, hence it is the most under-diagnosed and under-treated nutritional deficiency in the world [14-16]. The potential benefits of sufficient vitamin D levels are innumerable, including both skeletal and non-skeletal benefits and so more emphasis must be given to diagnosing and treating vitamin D deficiency, especially in the developing countries of the world. The aim of this study was to assess the serum vitamin D levels, identify the prevalence of insufficiency and deficiency of vitamin D and finally to explore and identify any gender or age bias can be identified in a hospital based population in Mumbai.

\section{Materials and Methods}

An approval from the institutional ethical committee of Nair Hospital Dental College was obtained and all the participants were explained the rationale of the study prior to the start of study. Patient's personal and demographic details were obtained along with an informed consent in the patient's language of convenience.

The study was carried out over a period of 21 months from June 2014 to march 2016. Since very few studies have been carried out on the population of Mumbai, a randomized sampling technique was utilized to select 100 adult patients who reported to Nair Hospital Dental College on an outpatient basis.

\section{Inclusion Criteria for Samples:}

- Patients from both the sexes.

- Patients in the age group of 25-70 years

- Patients with no known diagnosis that may affect the bones/ joints/ TMJ and undergoing routine blood investigations

\section{Exclusion Criteria:}

- Patients below 25 years of age and above 70 years of age.

- Patients having relative morbidity in terms of bone diseases etc.

- Patients who were unable to provide an informed consent.

Serum 25(OH)D levels of all the subjects were evaluated using chemiluminescence immunoassay (CLIA) technique. 25(OH)D levels below $10 \mathrm{ng} / \mathrm{mL}$ were categorized as "Deficiency" and between 29-11 ng/mL were categorized as "Insufficiency" of vitamin D.

\section{Results}

Of the 100 participants that took part in the study, 63 were female participants and 37 were male participants.

Only 49 subjects were identified with adequate vitamin D levels, 39 patients were identified to have vitamin D insufficiency $(<30 \mathrm{ng} / \mathrm{mL})$ while 13 patients were diagnosed with deficiency of vitamin D (Figure 1 and Table 1).

The results clearly showed that the incidence of both insufficiency and deficiency was higher in females as it can be seen in Figure 2,3 and 4 . 


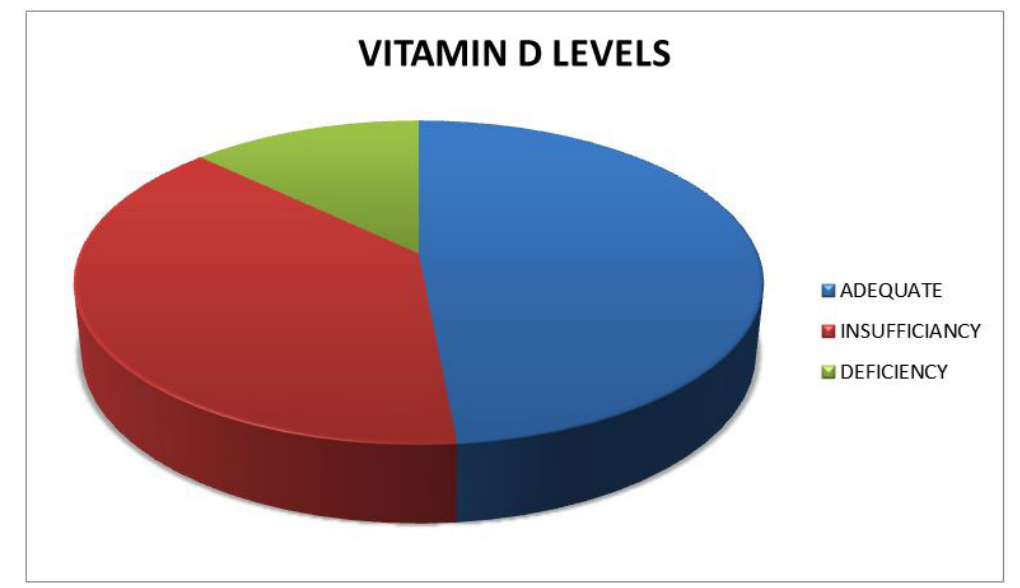

Figure 1: Incidence of Adequacy, Insufficiency and Deficiency of Vitamin D among participants

\begin{tabular}{|c|c|c|c|}
\hline & $\begin{array}{c}\text { INSUFFICIENCY } \\
(\mathbf{1 1 - 2 9} \mathbf{n g} / \mathbf{m L})\end{array}$ & $\begin{array}{c}\text { DEFICIENCY } \\
(<\mathbf{1 0} \mathbf{n g} / \mathbf{m L})\end{array}$ & TOTAL \\
\hline MALE & 15 & 5 & 37 \\
\hline FEMALE & 24 & 8 & 63 \\
\hline TOTAL & 39 & 13 & 100 \\
\hline
\end{tabular}

Table 1: Gender wise results of patients with insufficiency and deficiency of vitamin D

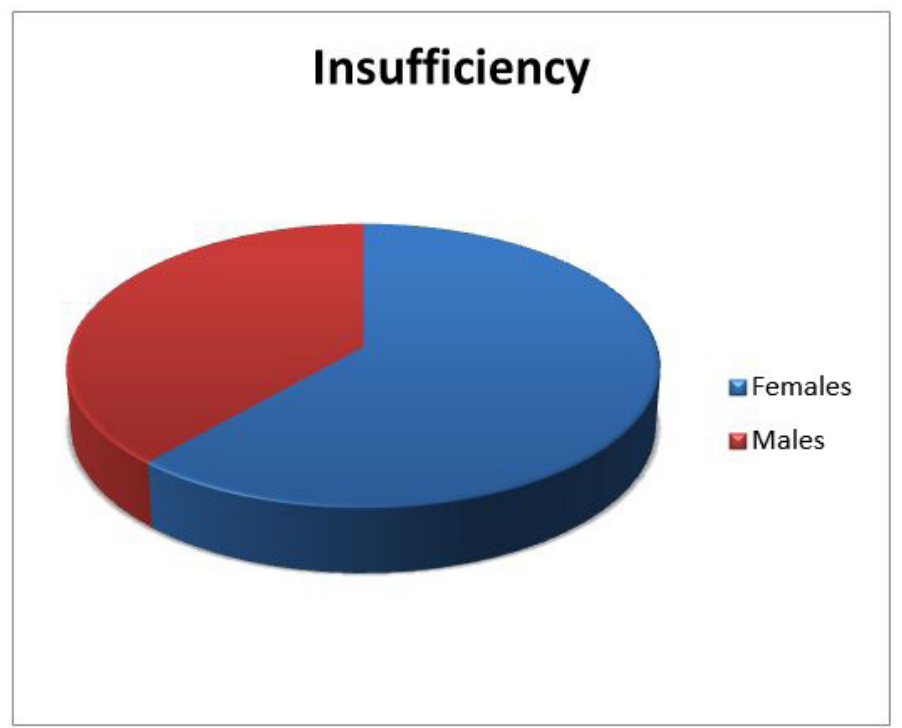

Figure 2: Gender distribution among participants with Insufficiency of Vitamin D

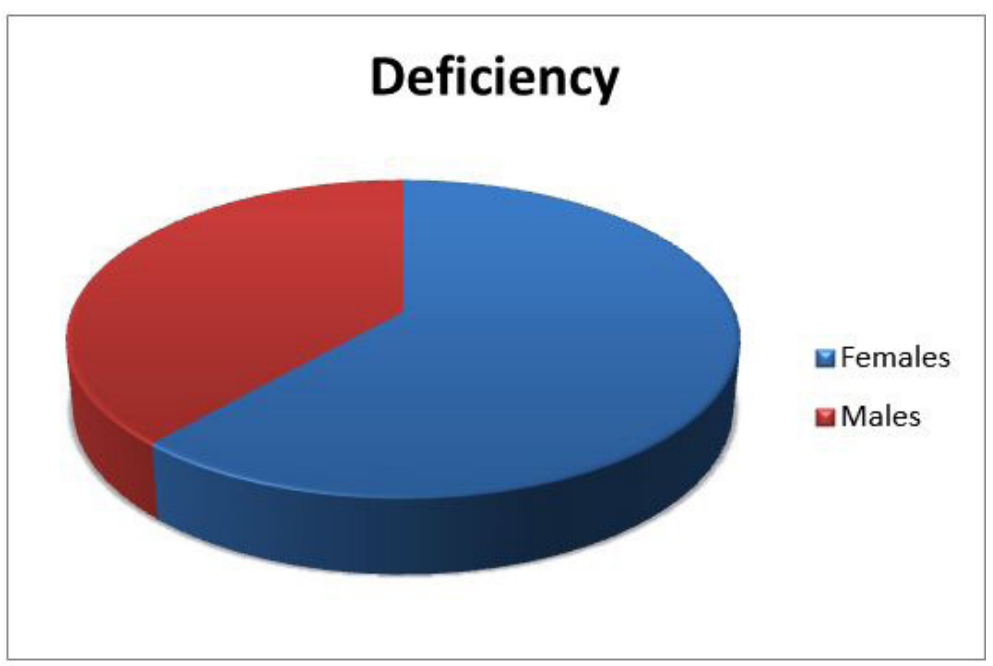

Figure 3: Gender distribution among participants with deficiency of Vitamin D 


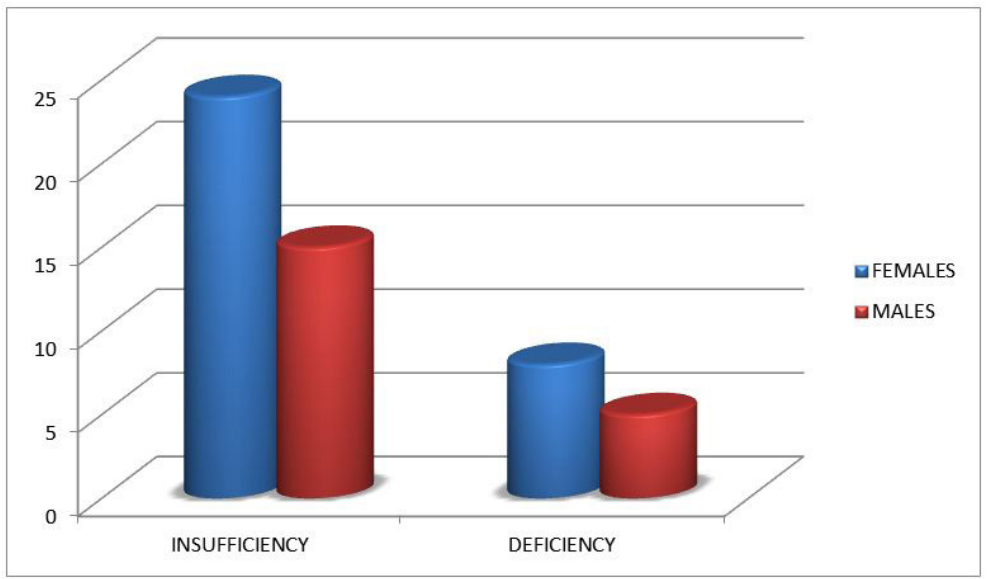

Figure 4: Incidence of Vitamin D Insufficiency $(25(\mathrm{OH}) \mathrm{D}<30 \mathrm{ng} / \mathrm{mL})$ and Deficiency $(25(\mathrm{OH}) \mathrm{D}<10 \mathrm{ng} / \mathrm{mL})$

The age distribution of the patients with vitamin D sufficiency, Insufficiency and Deficiency can be seen in Figure 5 . The incidence of vitamin D Insufficiency and deficiency was seen more in the higher age group. Most of the female subjects who were diagnosed with Vitamin D deficiency were above 40 years of age as can be seen in Figure 6.

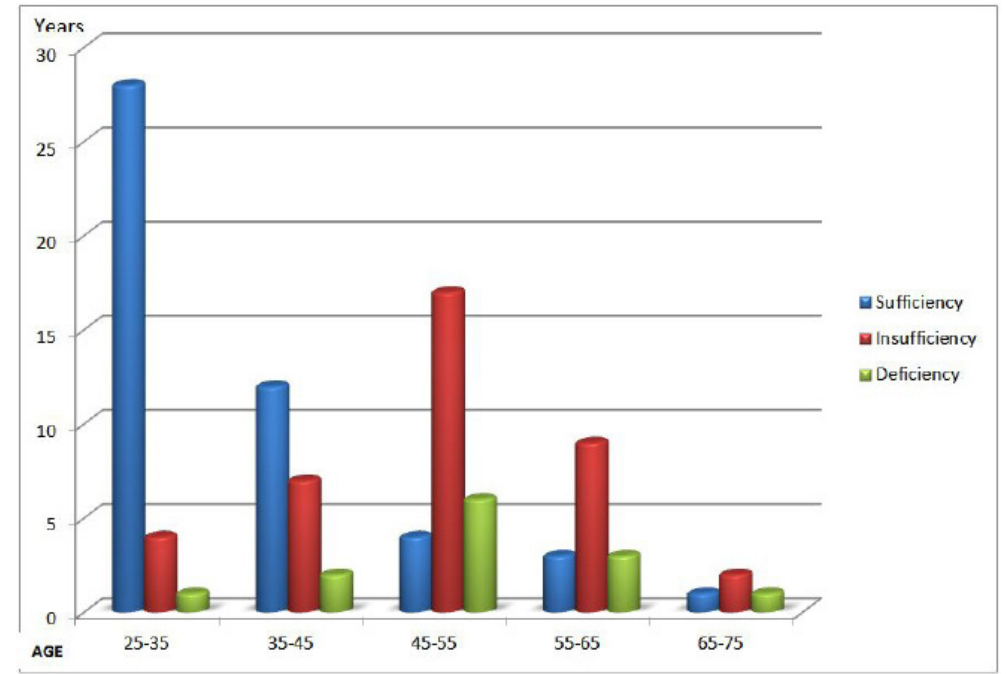

Figure 5: Age wise distribution of participants into Vitamin D Sufficiency, Insufficiency and Deficiency

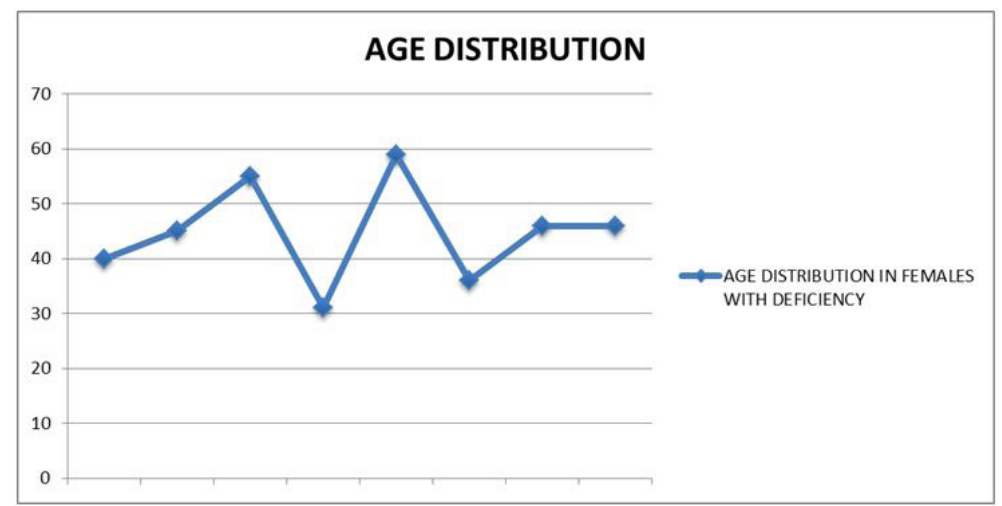

Figure 6: Age distribution of female subjects with vitamin D deficiency ( $25(\mathrm{OH}) \mathrm{D}<10 \mathrm{ng} / \mathrm{mL}$ )

\section{Discussion}

The 2 forms of Vitamin D are ergocalciferol (vitamin D2) and cholecalciferol (vitamin D3). Although Vitamin D can be obtained from the dietary animal sources like deep sea fatty fish, egg yolks, or liver and also from supplements, it is primarily produced in the skin in reaction to ultraviolet B radiation from sunlight. Very few plants sources contain Vitamin D2, but is commercially produced by irradiation of yeast which is used for fortification and supplementation [9]. Although various studies indicates that vitamin D2 may be metabolized more rapidly than vitamin D3, but they can be considered bioequivalent with regular daily intake [17-19]. However both forms D2 and D3 are biologically inactive until they undergo two enzymatic reactions. These are both 
hydroxylation reactions and the first of which occurs in the human liver. Cholecalciferol hydroxylates into 25-hydroxyvitamin D3 $[25(\mathrm{OH}) \mathrm{D}$, calcidiol] in liver. This is the most stable metabolite having a half-life of approximately 3 weeks because of which it is used to determine the vitamin $\mathrm{D}$ status. Further, $25(\mathrm{OH}) \mathrm{D}$ is hydroxylated to 1,25 -dihydroxyvitamin $\mathrm{D}[1,25(\mathrm{OH}) 2 \mathrm{D}]$ in the kidney. This is the biologically active form that aids calcium absorption in the duodenum. It acts at a cellular level on both the osteoblasts and osteoclasts, to mobilize calcium. A number of characteristics of Vitamin D are those of a steroid hormone and not that of a vitamin and so now it is considered as a prohormone rather than a true vitamin [9].

Although it becomes difficult to define the optimum value for Vitamin D, the 25(OH)D level needed to achieve skeletal health, as also optimal benefits on nonskeletal health outcomes is considered optimum. One of the method to functionally define the optimal vitamin D status is the $25(\mathrm{OH}) \mathrm{D}$ level that maximally suppresses PTH secretion. Multiple cross sectional examinations that explored the relationship between serum PTH and 25(OH)D levels demonstrated a plateau in suppression of PTH when the $25(\mathrm{OH}) \mathrm{D}$ level reaches approximately $30 \mathrm{ng} / \mathrm{mL}$ [20]. Another method used by some researchers to define the optimal vitamin D status is the $25(\mathrm{OH}) \mathrm{D}$ level at which after administration of vitamin $\mathrm{D}$, there is no further incremental increase in $1,25(\mathrm{OH}) 2 \mathrm{D}$ levels which was found to be 25 to $30 \mathrm{ng} / \mathrm{mL}$ [21]. A few studies have used the 25(OH)D level that results in maximal intestinal calcium absorption to define the optimal value for vitamin D. this was found to occur when the $25(\mathrm{OH}) \mathrm{D}$ levels were $32 \mathrm{ng} / \mathrm{mL}$ or greater [22]. Due to these multiple evidences in the current study we used 30ng/mL as the cut off value for adequacy. Values below $10 \mathrm{ng} / \mathrm{mL}$ were categorized as vitamin $\mathrm{D}$ deficiency and those between $10 \mathrm{ng} / \mathrm{mL}$ and $30 \mathrm{ng} / \mathrm{mL}$ were categorized as Insufficiency of vitamin $\mathrm{D}$.

The values of 25(OH)D vary considerably with the geography of the area, due to changing sun exposure and diets. However the values also vary with sex and age. In the study we found that more female participants were diagnosed with vitamin D insufficiency in comparison to male subjects. Similar results were observed in various studies carried out all over India. In the study carried out by Harinarayan in 2008 in Tirupati in southern India insufficiency and deficiency was seen higher in urban as well as rural adult females in comparison to their male counterparts [23]. In 2010, Multani carried out a study among resident doctors in Mumbai which also showed higher levels of vitamin D deficiency among female doctors [24].

In the current study we found that the female subjects who were diagnosed with vitamin D deficiency were of the higher age group; majority of the females were above 40 years of age. In the studies conducted by Harinarayan in 2005 and Paul in 2008 it was noted that post-menopausal women from rural and semi urban habitats in Tirupati in southern India had significantly higher rates of vitamin D deficiency [10,25].

Although India lies in the tropical region and is believed to have adequate sunlight around the year, it must be noted that Vitamin D sufficiency via sun exposure may not be a rational solution for most Indians. For adequacy, an individual's bare skin should be exposed to sunlight to photosynthesize vitamin D. Indians naturally have darker skin that has high melanin content. This melanin tends to acts as a natural sunscreen. Therefore, in comparison to individuals with fairer skin, such as Caucasians, darker skin produces a significantly lesser amount of vitamin D. Urbanization also has a part to play in causing vitamin D deficiency. Overcrowded living environment often prevents direct sunlight from reaching the homes in cities. Added to this is the atmospheric pollution of metropolitan cities, spending majority of the time at work indoors, the limited outdoor activities and the use of sunscreen and umbrellas to protect the skin from the scorching heat of the Indian sun [6-8,26,27]. The higher prevalence of Vitamin D deficiency in women can be attributed to many social customs prevalent in India like the use of pardah, enforced use of completely covering clothing and even social restrictions to going outdoors.

Vitamin D deficiency is known to affect 70\%-100\% of ostensibly healthy individuals in India. Vitamin D deficiency adversely affects the skeletal components and causes rickets in children and osteomalacia in adults. It is linked to various Oral diseases like increases caries susceptibility, predisposition to temporomandibular joint (TMJ) disorders and musculoskeletal pain [28]. The extra-skeletal ill effects of vitamin D deficiency are also innumerable. The female population is significantly more affected and this can have adverse effects on their pregnancy too. The quality of life is adversely affected in the geriatric population due to vitamin D deficiency [29]. Hence it is important that awareness should be created about adequate sun exposure to avoid deficiency. Supplementation and fortification of food may be considered a viable option when adequate exposure to sun is not possible however its feasibility may be in question. The small sample size and randomized sampling were the limitations of this study and more research must be carried out to identify the incidence of vitamin D Insufficiency and deficiency and strategies to tackling this problem.

\section{Conclusion}

The primary source of vitamin D remains exposure of skin to the sunlight and not dietary sources. In spite of Mumbai lying in the latitudes that offer adequate exposure to sunlight, $52 \%$ of the subjects in the study did not have adequate vitamin $\mathrm{D}$ levels. Urbanization, overcrowding, lifestyle changes like lack of outdoor activities along with rising pollution can be linked to this increasing vitamin D deficiency in cities. Females were found to be affected more by vitamin D deficiency and insufficiency and the cause can be traced back to a lot of social customs that restrict the exposure of "bare skin" to sunlight. With more and more research indicating the importance of Vitamin D for skeletal and extra-skeletal health efforts should be directed not only to identifying this problem but also finding rational and feasible solutions to treating vitamin D deficiency and insufficiency in the Indian population. 


\section{Acknowledgement}

We would like to express our deepest gratitude to the publishers of Journal of Nutrition and Health Sciences, Annex Publishers, Manassas, Virginia, USA for providing full publication support for this work.

\section{References}

1. Holick MF, Chen TC (2008) Vitamin D deficiency: a worldwide problem with health consequences. Am J Clin Nutr 87: 1080-6.

2. Anderson PG, Williams CHM, Halderson H, Summerfeldt C, Agnew RG (1934) The Influence of Vitamin D in the Prevention of Dental Caries. J Am Dent Assoc 21: 1349-66.

3. Dietrich T, Joshipura KJ, Dawson-Hughes B, Bischoff-Ferrari HA (2004) Association between serum concentrations of 25-hydroxyvitamin D3 and periodontal disease in the US population. Am J Clin Nutr 80: 108-13.

4. Krall EA, Wehler C, Garcia RI, Harris SS, Dawson-Hughes B (2001) Calcium and vitamin D supplements reduce tooth loss in the elderly. Am J Med 111: 452-6. 5. Holick MF (2010) The functional metabolism and molecular biology of vitamin D action In: Vitamin D: Physiology, Molecular Biology, and Clinical Applications $2^{\text {nd }}$ Ed. Humana Press, New York 61-97.

6. Ritu G, Gupta A (2014) Vitamin D deficiency in India: prevalence, causalities and interventions. Nutrients 6: 729-75.

7. Clemens TL, Adams JS, Henderson SL, Holick MF (1982) Increased skin pigment reduces the capacity of skin to synthesise vitamin D3. Lancet 1: 74-6.

8. Matsuoka LY, Wortsman J, Haddad JG, Kolm P, Hollis BW (1991) Racial pigmentation and the cutaneous synthesis of vitamin D. Arch Dermatol 127: 536-8.

9. Thacher TD, Clarke BL (2011) Vitamin D Insufficiency. Mayo Clin Proc 86: 50-60.

10. Harinarayan CV (2005) Prevalence of vitamin D insufficiency in postmenopausal south Indian women. Osteoporos Int 16: 397-402.

11. Shivane VK, Sarathi V, Bandgar T, Menon P, Shah NS (2011) High prevalence of hypovitaminosis D in young healthy adults from the western part of India. Postgrad Med J 87: 514-8.

12. Sahu M, Das V, Aggarwal A, Rawat V, Saxena P, et al. (2009) Vitamin D replacement in pregnant women in rural north India: a pilot study. Eur J Clin Nutr 63: $1157-9$.

13. Harinarayan CV, Joshi SR (2009) Vitamin D status in India--its implications and remedial measures. J Assoc Physicians India 57: 40-8.

14. van Schoor NM, Lips P (2011) Worldwide vitamin D status. Best Pract Res Clin Endocrinol Metab 25: 671-80.

15. Mithal A, Wahl DA, Bonjour JP, Burckhardt P, Dawson-Hughes B, et al. (2009) Global vitamin D status and determinants of hypovitaminosis D. Osteoporos Int 20: 1807-20.

16. van der Meer IM, Middelkoop BJ, Boeke AJ, Lips P (2011) Prevalence of vitamin D deficiency among Turkish, Moroccan, Indian and sub-Sahara African populations in Europe and their countries of origin: an overview. Osteoporos Int 22: 1009-21.

17. Armas LA, Hollis BW, Heaney RP (2004) Vitamin D2 is much less effective than vitamin D3 in humans. J Clin Endocrinol Metab 89: 5387-91.

18. Trang HM, Cole DE, Rubin LA, Pierratos A, Siu S, et al. (1998) Evidence that vitamin D3 increases serum 25-hydroxyvitamin D more efficiently than does vitamin D2. Am J Clin Nutr 68: 854-8.

19. Holick MF, Biancuzzo RM, Chen TC, Klein EK, Young A, et al. (2008) Vitamin D2 is as effective as vitamin D3 in maintaining circulating concentrations of 25-hydroxyvitamin D. J Clin Endocrinol Metab 93: 677-81.

20. Chapuy MC, Preziosi P, Maamer M, Arnaud S, Galan P, et al. (1997) Prevalence of vitamin D insufficiency in an adult normal population. Osteoporos Int 7: $439-43$.

21. Thacher TD, Obadofin MO, O'Brien KO, Abrams SA (2009) The effect of vitamin D2 and vitamin D3 on intestinal calcium absorption in Nigerian children with rickets. J Clin Endocrinol Metab 94: 3314-21.

22. Heaney RP (2004) Functional indices of vitamin D status and ramifications of vitamin D deficiency. Am J Clin Nutr 80: 1706S-9S.

23. Harinarayan CV, Ramalakshmi T, Prasad UV, Sudhakar D (2008) Vitamin D status in Andhra Pradesh : a population based study. Indian J Med Res 127: 211-8.

24. Multani SK, Sarathi V, Shivane V, Bandgar TR, Menon PS, et al. (2010) Study of bone mineral density in resident doctors working at a teaching hospital. J Postgrad Med 56: 65-70.

25. Paul TV, Thomas N, Seshadri MS, Oommen R, Jose A, et al. (2008) Prevalence of osteoporosis in ambulatory postmenopausal women from a semiurban region in Southern India: relationship to calcium nutrition and vitamin D status. Endocr Pract 14: 665-71.

26. Lo CW, Paris PW, Holick MF (1986) Indian and Pakistani immigrants have the same capacity as Caucasians to produce vitamin D in response to ultraviolet irradiation. Am J Clin Nutr 44: 683-5.

27. Agarwal KS, Mughal MZ, Upadhyay P, Berry JL, Mawer EB, et al. (2002) The impact of atmospheric pollution on vitamin D status of infants and toddlers in Delhi, India. Arch Dis Child 87: 111-3.

28. Khanna S, Gharpure AS (2014) Vitamin D3: Unearthing the Ancient Elixir. J Community Nutr Health 2: 52-5.

29. Jagur O, Kull M, Leibur E, Kallikorm R, Loorits D, et al. (2011) Relationship between radiographic changes in the temporomandibular joint and bone mineral density: a population based study. Stomatologija 13: 42-8. 


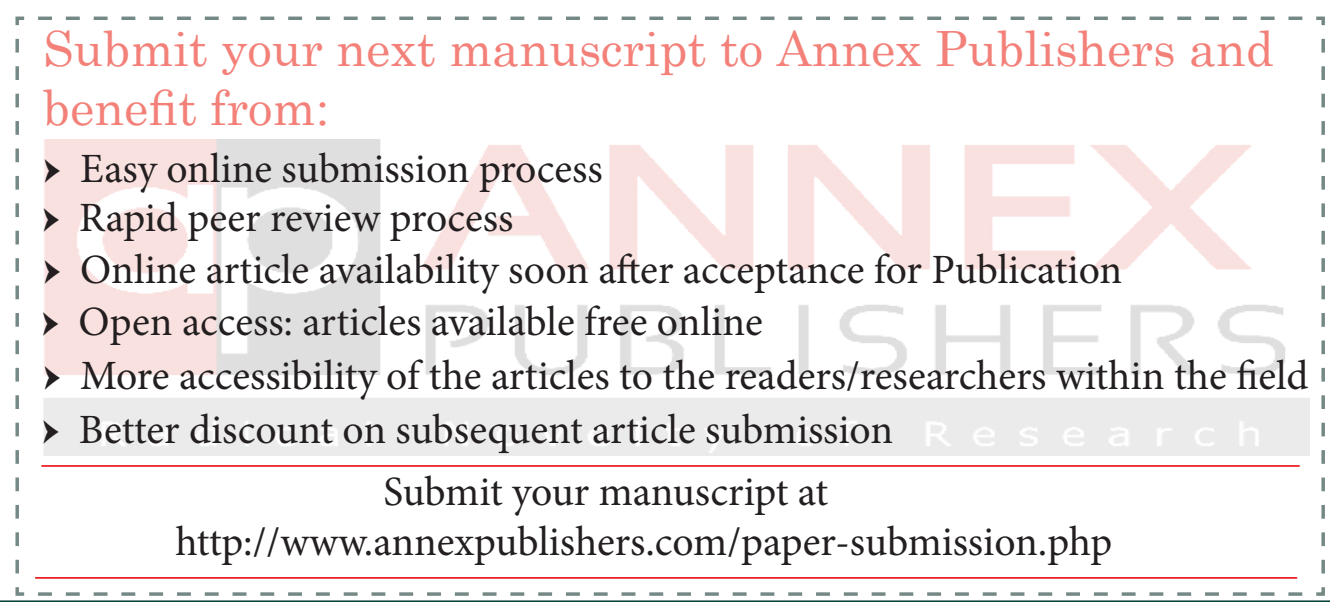

